\title{
Saddle Point Problems, Bilevel Problems, and Mathematical Program with Equilibrium Constraint on Complete Metric Spaces
}

\author{
Lai-Jiu Lin and Chih-Sheng Chuang \\ Department of Mathematics, National Changhua University of Education, Changhua 50058, Taiwan
}

Correspondence should be addressed to Lai-Jiu Lin, maljlin@cc.ncue.edu.tw

Received 4 November 2009; Accepted 15 January 2010

Academic Editor: Yeol Je Cho

Copyright (C) 2010 L.-J. Lin and C.-S. Chuang. This is an open access article distributed under the Creative Commons Attribution License, which permits unrestricted use, distribution, and reproduction in any medium, provided the original work is properly cited.

We apply an existence theorem of variational inclusion problem on metric spaces to study optimization problems, set-valued vector saddle point problems, bilevel problems, and mathematical programs with equilibrium constraint on metric spaces. We study these problems without any convexity and compactness assumptions. Our results are different from any existence results of these types of problems in topological vector spaces.

\section{Introduction}

Let $\left(X, d_{X}\right)$ and $\left(Y, d_{Y}\right)$ be two metric spaces, let $Z$ be a real Hausdorff topological vector space ordered by a nonempty pointed closed convex cone $K$ in $Z$ with nonempty interior, and let $W$ be a real Banach space ordered by a nonempty pointed closed convex cone $C$ with nonempty interior. Let $G: X \times Y \multimap W, S: X \times Y \times X \multimap Z$, and $P: X \times Y \multimap Z$ be multivalued maps. Throughout this paper, we use these notations unless specified otherwise. In this paper, the following vector mathematical programs with equilibrium constraint on metric spaces are considered.

(MPEC-1) $\operatorname{Min}_{C} G(x, y)$ is subject to $(x, y) \in X \times Y$, and $S(x, y, u) \cap[K \backslash\{0\}]=\emptyset$ for all $u \in X$.

(MPEC-2) $\operatorname{Min}_{C} G(x, y)$ is subject to $(x, y) \in X \times Y$, and $S(x, y, u) \nsubseteq K \backslash\{0\}$ for all $u \in X$.

If $Z=\mathbb{R}, K=[0, \infty)$, and $S$ is a real function, then problems (MPEC-1) and (MPEC-2) are reduced to the following problem:

(MPEC-3) $\operatorname{Min}_{C} G(x, y)$ is subject to $(x, y) \in X \times Y$, and $S(x, y, u) \leq 0$ for all $u \in X$. 
We also study the following bilevel problems.

(BL-1) $\operatorname{Min}_{C} G(x, y)$ is subject to $(x, y) \in X \times Y$, for $y \in Y, x$ is a solution of problem $Q_{1}(y): W_{M i n} P(x, y)$ (i.e., for $y \in Y, P(x, y) \cap \operatorname{WMin}_{K} P(X, y) \neq \emptyset$ ), for $x \in X$, and $y$ is a solution of problem $R_{1}(x)$ : $\operatorname{WMax}_{K} P(x, y)$. (i.e., for $x \in X, P(x, y) \cap$ $\left.\mathrm{WMax}_{K} P(x, Y) \neq \emptyset\right)$.

(BL-2) $\operatorname{Min}_{c} G(x, y)$ is subject to $(x, y) \in X \times Y, P(x, y) \nsubseteq P(a, y)+\operatorname{int}(K)$ for all $a \in X$, and $P(x, b) \nsubseteq P(x, y)+\operatorname{int}(K)$ for all $b \in Y$.

(BL-3) $\operatorname{Min}_{c} G(x, y)$ is subject to $(x, y) \in X \times Y$, for $x \in X, y$ is a solution of problem $Q_{2}(x): \operatorname{Min}_{\mathbb{R}_{+}^{m}} P(x, y)$ and (i.e., for $\left.x \in X, P(x, y) \cap \operatorname{Min}_{\mathbb{R}_{+}^{m}} P(x, Y) \neq \emptyset\right)$, for $y \in$ $Y, x$ is a solution of problem $R_{2}(y)$ : $\operatorname{Max}_{\mathbb{R}_{+}^{m}} P(x, y)$. (i.e., for $y \in Y, P(x, y) \cap$ $\left.\operatorname{Max}_{\mathbb{R}_{+}^{m}} P(X, y) \neq \emptyset\right)$. problem.

If $P: X \times Y \rightarrow Z$ is a single valued function, then (BL-2) is reduced to the following

(BL-4) $\operatorname{Min}_{C} G(x, y)$ is subject to $(x, y) \in X \times Y$, for $y \in Y$, and $x$ is a solution of problem and $Q_{3}(y)$ : $\operatorname{WMin}_{K} P(x, y)$; and for $x \in X, y$ is a solution of problem $R_{3}(x)$ : $\mathrm{WMax}_{K} P(x, y)$.

Problem (BL-1) has applications in real world. Let $X$ be the set of government's agricultural policies and let $Y$ be the set of government's industrial policies. For each $x \in X$ and $y \in Y$, let $G(x, y)$ be the amount of money that the government uses to promote agriculture and industrial developments, and let $P(x, y)$ be the degree of industrial development. We suppose that as the industry becomes more and more developed, the losses from the agriculture sector rise accordingly. Therefore, the solution of problem (BL1) represents the government's best policy to promote the development of the industry so that the losses from agriculture sector will be as minimal as possible, while the amount of money that the government uses to promote the policies can be the lowest possible.

Mathematical program with equilibrium constraint and bilevel problem represent two important classes of optimization problems which have been investigated in a large number of articles and books. We find in the literatures that Luo et al. [1], Stein and Still [2], Stein [3], Birbil et al. [4], Liou et al. [5], Lin and Still [6], Lin [7], as well asLin and Hsu [8] have studied mathematical program with equilibrium constraint and bilevel problem on topological vector spaces. As usual in linear and nonlinear optimization, these studies mainly deal with optimality conditions and numerical methods to solve these problems and typically the existence of feasible points is tacitly assumed. Besides, the domains of the functions they consider are subsets of topological vector spaces and certain convexity assumptions on the functions are needed. In this paper, we study these problems with functions defined on metric spaces, so we do not need any convexity assumptions on the functions we consider. We study the existence theorems of solutions for (MPEC-1), (MPEC-2), (BL-1), (BL-2), and (BL-3). To the best of our knowledge, there is no result of these types of problems on metric space. problems.

In this paper, we also study the following loose set-valued vector saddle point

(LSP-1) For each $(u, v) \in X \times Y$, find $(\bar{x}, \bar{y}) \in X \times Y$ such that $P(u, \bar{y}) \cap[P(\bar{x}, v)+K] \neq \emptyset$, $P(\bar{x}, \bar{y}) \cap \operatorname{Min}_{K} P(X, \bar{y}) \neq \emptyset$, and $P(\bar{x}, \bar{y}) \cap \operatorname{Max}_{K} P(\bar{x}, Y) \neq \emptyset$.

(LSP-2) For each $(u, v) \in X \times Y$, find $(\bar{x}, \bar{y}) \in X \times Y$ such that $P(u, \bar{y}) \subseteq P(\bar{x}, v)+K$, $P(\bar{x}, \bar{y}) \nsubseteq P(x, \bar{y})+K$, and $P(\bar{x}, y) \nsubseteq P(\bar{x}, \bar{y})+K$ for all $x \in X \backslash\{\bar{x}\}$ and all $y \in Y \backslash\{\bar{y}\}$. 
If $P: X \times Y \rightarrow Z$ is a map, then problems (LSP-1) and (LSP-2) are reduced to the following vector saddle point problems (VSP).

(i) Find $(\bar{x}, \bar{y}) \in X \times Y$ such that $P(\bar{x}, \bar{y}) \in \operatorname{Min}_{K} P(X, \bar{y}) \cap \operatorname{Max}_{K} P(\bar{x}, Y)$.

There are many results on loose saddle point problems, vector saddle point, and saddle point problems (see, e.g., [9-17]). But to the best of our knowledge, there are no existence theorems in the literatures for loose saddle point and vector saddle point problems for functions defined on the product of metric spaces. We study the loose saddle point problems and vector saddle point problems for functions defined on the product of metric spaces. We do not assume any compact assumptions on the spaces and convexity assumptions on the maps we consider. As for applications of our existence theorems on saddle point problems, we study bilevel problems on metric spaces. We also study mathematical programs with equilibrium constraint. Our results on mathematical programs with equilibrium constraint, bilevel problems, loose saddle point problems, and vector saddle point problems are different from any existence results of these types of problems in the literatures.

\section{Preliminaries}

Let $X$ and $Y$ be topological spaces (in short t.s.), $T: X \multimap Y$ be a multivalued map. $T$ is said to be u.s.c. (resp., l.s.c.) at $x \in X$ if for every open set $U$ in $Y$ with $T(x) \subseteq U$ (resp., $T(x) \cap U \neq \emptyset$ ), there exists an open neighborhood $V$ of $x$ such that $T\left(x^{\prime}\right) \subseteq U$ (resp., $T\left(x^{\prime}\right) \cap U \neq \emptyset$ ) for all $x^{\prime} \in V ; T$ is said to be u.s.c. (resp., l.s.c.) on $X$ if $T$ is u.s.c. (resp., l.s.c.) at every point of $X$; $T$ is continuous at $x$ if $T$ is both u.s.c. and l.s.c. at $x ; T$ is said to be closed $\operatorname{if} \operatorname{Gr}(T)=\{(x, y) \in$ $X \times Y: y \in T(x), x \in X\}$ is a closed set in $X \times Y$; $T$ is said to be open if $\operatorname{Gr}(T)$ is an open set in $X \times Y$. For a subset $A$ of topological space $X$, let $\mathrm{cl}(A)$ denote the closure of $A$.

Lemma 2.1 (see [18]). Let $X$ and $Y$ be topological spaces, and let $T: X \multimap Y$ be a multivalued map. Then $T$ is l.s.c. at $x \in X$ if and only if for any $y \in T(x)$ and any net $\left\{x_{\alpha}\right\}_{\alpha \in \Lambda}$ in $X$ converges to $x$, there exists a net $\left\{y_{\alpha}\right\}_{\alpha \in \Lambda}$ such that $y_{\alpha} \in T\left(x_{\alpha}\right)$ for all $\alpha \in \Lambda$ and $y_{\alpha} \rightarrow y$.

Lemma 2.2 (see [19]). Let $X$ and $Y$ be Hausdorff topological spaces, and let $T: X \multimap Y$ be a multivalued map. (i) If $T$ is an u.s.c. multivalued map with nonempty closed values, then $T$ is closed; (ii) if $\mathrm{X}$ is a compact set and $T$ is an u.s.c. multivalued map with nonempty compact values, then $T(X)$ is compact.

Definition 2.3. Let $A$ be a nonempty subset of a t.v.s. $Z$ ordered by a nonempty pointed closed convex cone $K$. An element $a \in A$ is said to be a minimal (resp., maximal) point of $A$ if $A \cap(a-K)=\{a\}$ (resp., $A \cap(a+K)=\{a\}$ ). Here, $\operatorname{Min}_{K} A$ and $\operatorname{Max}_{K} A$ denote the sets of minimal point of $A$ and maximal point of $A$, respectively.

Definition 2.4. Let $A$ be a nonempty subset of a t.v.s. $Z$ ordered by a nonempty pointed closed convex cone $K$ with nonempty interior. An element $a \in A$ is said to be a weakly minimal (resp., weakly maximal) point of $A$ if $A \cap(a-\operatorname{int}(K))=\emptyset$ (resp., $A \cap(a+\operatorname{int}(K))=\emptyset)$. Here, $\mathrm{WMin}_{K} A$ and $\mathrm{WMax}_{K} A$ denote the sets of weakly minimal point of $A$ and weakly maximal point of $A$, respectively.

Theorem 2.5 (see [20]). Let $Z$ be a Hausdorff t.v.s. ordered by a nonempty pointed closed convex cone $K$ with nonempty interior. If $A$ is a nonempty compact subset of $Z$, then $\operatorname{Min}_{K} A \neq \emptyset$ and $\operatorname{Max}_{K} A \neq \emptyset$. 
Definition 2.6 (see [21]). Let $K$ be pointed closed convex cone with nonempty interior in a Banach space $Z$. Then $K$ is called normal if there exists $\lambda>0$ such that if $x, y \in K$ and $y-x \in K$, then $\|x\| \leq \lambda\|y\|$. Here, it is called $\lambda$-normal pointed closed convex cone.

Theorem 2.7 (see [22]). Let $\left(X, d_{X}\right)$ be a complete metric space, and let $Z$ be a Hausdorff t.v.s.. Let $F: X \times X \multimap Z$ be a multivalued map. Assume that

(i) $0 \in F(x, x)$ for each $x \in X$,

(ii) for each $x \in X,\{y \in X: 0 \in F(x, y)\}$ is a closed subset of $X$,

(iii) for each $x, y, z \in X$, if $0 \in F(x, y)$ and $0 \in F(y, z)$, then $0 \in F(x, z)$,

(iv) for each sequence $\left\{x_{n}\right\}_{n \in \mathbb{N}}$ in $X$, if $0 \in F\left(x_{n}, x_{n+1}\right)$, then $d_{X}\left(x_{n}, x_{n+1}\right) \rightarrow 0$ as $n \rightarrow \infty$.

Then there exists $\bar{x} \in X$ such that $0 \notin F(\bar{x}, y)$ for all $y \in X \backslash\{\bar{x}\}$.

Theorem 2.8. Let $\left(X, d_{X}\right)$ be a complete metric space. Let $G: X \multimap W$ be a multivalued map with nonempty values. Assume that

(i) $G$ is closed,

(ii) for each sequence $\left\{\left(x_{n}, y_{n}\right)\right\}_{n \in \mathbb{N}}$ in $X \times W$, if $y_{n} \in G\left(x_{n}\right)$ and $y_{n}-y_{n+1} \in C$ for all $n \in \mathbb{N}$, then $d_{X}\left(x_{n}, x_{n+1}\right) \rightarrow 0$ and $\left\|y_{n}-y_{n+1}\right\| \rightarrow 0$ as $n \rightarrow \infty$.

Then there exists $\bar{x} \in X$ such that $G(\bar{x}) \cap \operatorname{Min}_{C} G(X) \neq \emptyset$.

Proof. Let $k_{0} \in C \backslash\{0\}$ be fixed. Clearly, $\operatorname{Gr}(G)$ is a complete metric space. Let $F$ : $\operatorname{Gr}(G) \times$ $\operatorname{Gr}(G) \multimap \mathbb{R}$ be defined by $F\left(\left(x_{1}, y_{1}\right),\left(x_{2}, y_{2}\right)\right)=\left\{t \in \mathbb{R}: y_{1}-y_{2}+t k_{0} \in C\right\}$ for each $\left(x_{1}, y_{1}\right),\left(x_{2}, y_{2}\right) \in \operatorname{Gr}(G)$. Clearly, $0 \in F((x, y),(x, y))$ for each $(x, y) \in \operatorname{Gr}(G)$. For each $(x, y) \in \operatorname{Gr}(G)$, we know that

$$
\{(u, v) \in \operatorname{Gr}(G): 0 \in F((x, y),(u, v))\}=\{(u, v) \in \operatorname{Gr}(G): y-v \in C\} .
$$

If $\left\{\left(u_{n}, v_{n}\right)\right\}_{n \in \mathbb{N}}$ is a sequence in $\{(u, v) \in \operatorname{Gr}(G): 0 \in F((x, y),(u, v))\}$ and $\left(u_{n}, v_{n}\right) \rightarrow$ $(u, v)$ as $n \rightarrow \infty$, then $\left(u_{n}, v_{n}\right) \in \operatorname{Gr}(G)$ and $y-v_{n} \in C$ for all $n \in \mathbb{N}$. Clearly, $y-v \in C$ and $(u, v) \in \operatorname{Gr}(G)$. By (ii), condition (iv) of Theorem 2.7 is satisfied. By Theorem 2.7, there exists $(\bar{x}, \bar{y}) \in \operatorname{Gr}(G)$ such that $\bar{y}-y \notin C$ for all $(x, y) \in \operatorname{Gr}(G) \backslash\{(\bar{x}, \bar{y})\}$. Clearly, $\bar{y} \in G(X) \cap(\bar{y}-C)$. If $y \in G(X) \cap(\bar{y}-C)$ and $y \neq \bar{y}$, then there exists $x \in X$ such that $y \in G(x)$ and $\bar{y}-y \in C$. Then $(x, y) \in \operatorname{Gr}(G) \backslash\{(\bar{x}, \bar{y})\}$ and this implies that $\bar{y}-y \notin C$. This leads to a contradiction. Hence, $G(X) \cap(\bar{y}-C)=\{\bar{y}\}$. This implies that $G(\bar{x}) \cap \operatorname{Min}_{C} G(X) \neq \emptyset$.

Example 2.9. Let $W=\mathbb{R}, X=(-\infty, 0] \cup\{1\}, C=[0, \infty)$, and $k_{0}=1$. Let $G: X \multimap W$ be defined by $G(x):=\{-x\}$ for each $x \in X$. Then by Theorem 2.8 , there exists $\bar{x} \in X$ such that $G(\bar{x}) \cap \operatorname{Min}_{c} G(X) \neq \emptyset$.

Example 2.10. Let $W=X=\mathbb{R}, C=[0, \infty)$, and $k_{0}=1$. Let $G: X \multimap W$ be defined by $G(x):=\{-x\}$ for each $x \in X$. Clearly, $G$ is closed. But condition (ii) of Theorem 2.8 does not hold. Indeed, let $\left\{\left(x_{n}, y_{n}\right)\right\}=\{(n,-n)\}_{n \in \mathbb{N}}$. Then $y_{n} \in G\left(x_{n}\right)$ for all $n \in \mathbb{N}$, and $d_{X}\left(x_{n}, x_{n+1}\right)=1$ and $\left\|y_{n}-y_{n+1}\right\|=1$ for all $n \in \mathbb{N}$. Furthermore, there is no $\bar{x} \in X$ such that $G(\bar{x}) \cap \operatorname{Min}_{c} G(X) \neq \emptyset$. Hence, condition (ii) of Theorem 2.8 is essential in Theorem 2.8. 
Theorem 2.11. Let $X$ be a metric space. Let $G: X \multimap W$ be a multivalued map with nonempty values. Assume that

(i) $G(X)$ is a nonempty closed subset of $W$,

(ii) for each sequence $\left\{y_{n}\right\}_{n \in \mathbb{N}}$ in $G(X)$, if $y_{n}-y_{n+1} \in C$, then $\left\|y_{n}-y_{n+1}\right\| \rightarrow 0$ as $n \rightarrow \infty$.

Then there exists $\bar{x} \in X$ such that $G(\bar{x}) \cap \operatorname{Min}_{c} G(X) \neq \emptyset$.

Proof. Let $k_{0} \in C \backslash\{0\}$ be fixed. Clearly, $G(X)$ is a complete metric space. Let $F: G(X) \times$ $G(X) \multimap \mathbb{R}$ be defined by $F\left(y_{1}, y_{2}\right)=\left\{t \in \mathbb{R}: y_{2}-y_{1}-t k_{0} \in-C\right\}$ for each $y_{1}, y_{2} \in G(X)$. Clearly, $0 \in F(y, y)$ for each $y \in G(X)$. Besides, for each $y_{1} \in G(X)$, let $A_{y_{1}}:=\{y \in G(X): 0 \in$ $\left.F\left(y_{1}, y\right)\right\}$. Then $A_{y_{1}}=\left\{y \in G(X): y-y_{1} \in-C\right\}$ is a closed set. Next, for each $y_{1}, y_{2}, y_{3} \in G(X)$, if $0 \in F\left(y_{1}, y_{2}\right)$ and $0 \in F\left(y_{2}, y_{3}\right)$, then $0 \in F\left(y_{1}, y_{3}\right)$. By (ii), for each sequence $\left\{y_{n}\right\}_{n \in \mathbb{N}}$ in $G(X)$ with $0 \in F\left(y_{n}, y_{n+1}\right)$, we have $\left\|y_{n}-y_{n+1}\right\| \rightarrow 0$ as $n \rightarrow \infty$. By Theorem 2.7, there exists $\bar{x} \in X$ such that $\bar{y} \in G(\bar{x}) \cap \operatorname{Min}_{c} G(X) \neq \emptyset$.

Example 2.12. Let $E=W=\mathbb{R}, X=\{-1\} \cup(0, \infty), C=[0, \infty)$, and $k_{0}=1$. Let $G: X \multimap W$ be defined by

$$
G(x):= \begin{cases}\{1,2\} & \text { if } x=-1 \\ \left\{2^{x}, 4\right\} & \text { if } x \in(0, \infty)\end{cases}
$$

Thus by Theorem 2.11, there exists $\bar{x} \in X$ such that $G \bar{x}) \cap \operatorname{Min}_{c} G(X) \neq \emptyset$. Note that $X$ is not closed, not open, and not convex.

Example 2.13. Let $X=\{1,2\}, W=\mathbb{R}^{2}$, and $C=\mathbb{R}_{+}^{2}$. Let $G: X \multimap W$ be defined by

$$
G(x):= \begin{cases}\left\{\left(y_{1}, y_{2}\right) \in \mathbb{R}^{2}: y_{1}=1,0 \leq y_{2} \leq 2\right\} & \text { if } x=1 \\ \left\{\left(y_{1}, y_{2}\right) \in \mathbb{R}^{2}: y_{1}=2,0 \leq y_{2} \leq 1\right\} & \text { if } x=2 .\end{cases}
$$

By Theorem 2.11, there exists $\bar{x} \in X$ such that $G(\bar{x}) \cap \operatorname{Min}_{c} G(X) \neq \emptyset$. Indeed, $\bar{x}=1$.

Remark 2.14. Example 2.10 also shows that condition (ii) of Theorem 2.11 is essential in Theorem 2.11. Next, the following result is a special case of Theorem 2.11. Note that it is different from Theorem 2.5 since we do not assume that $A$ is a compact set, but we assume that $W$ is a Banach space.

Corollary 2.15. Let $A$ be a nonempty closed subset of $W$. Suppose that for each sequence $\left\{y_{n}\right\}_{n \in \mathbb{N}}$ in $A$ if $y_{n}-y_{n+1} \in C$, then $\left\|y_{n}-y_{n+1}\right\| \rightarrow 0$ as $n \rightarrow \infty$. Then $\operatorname{Min}_{c} A \neq \emptyset$.

Proof. Let $X$ be a singleton subset of $W$. Then $X$ is a complete metric space. Let $G: X \multimap W$ be defined by $G(x)=A$ for each $x \in X$. By Theorem 2.11, there exists $\bar{x} \in X$ such that $G(\bar{x}) \cap \operatorname{Min}_{c} G(X) \neq \emptyset$ and this implies that $\operatorname{Min}_{c} A \neq \emptyset$.

Example 2.16. Let $W=\mathbb{R}^{2}$ and $A=C=\mathbb{R}_{+}^{2}$. It is easy to see that all conditions of Corollary 2.15 are satisfied. Hence, $\operatorname{Min}_{c} A \neq \emptyset$. Indeed, $\operatorname{Min}_{c} A=\{(0,0)\}$. Note that $A$ is not a compact subset of $W$. 
Corollary 2.17. Let $W$ be a Banach space, and let $C$ be a $\lambda$-normal pointed closed convex cone with nonempty interior $\lambda \in(0,1)$. If $A$ is a nonempty bounded closed subset of $W$, then $\operatorname{Min}_{c} A \neq \emptyset$.

Proof. Take any sequence $\left\{y_{n}\right\}_{n \in \mathbb{N}}$ in $A$ with $y_{n}-y_{n+1} \in C$. This implies that $\left(y_{n-k}-y_{n+1}\right)-$ $\left(y_{n-k-1}-y_{n+1}\right) \in C, k=1,2, \ldots,(n-2)$. By assumption, we get

$$
\left\|y_{n}-y_{n+1}\right\| \leq \lambda\left\|y_{n-1}-y_{n+1}\right\| \leq \cdots \leq \lambda^{n-1}\left\|y_{1}-y_{n+1}\right\|
$$

Since $A$ is bounded, there exists $M>0$ such that $\left\|y_{n}-y_{n+1}\right\| \leq \lambda^{n-1} M$ for all $n \in \mathbb{N}$. This implies that $\left\|y_{n}-y_{n+1}\right\| \rightarrow 0$ as $n \rightarrow \infty$ and Corollary 2.17 follows from Corollary 2.15.

Remark 2.18. Theorem 2.11 and Corollary 2.15 are equivalent.

Remark 2.19. From the above results and examples, we observe that if $A \subseteq \mathbb{R}^{m}$ is a closed set and there exists $a \in \mathbb{R}$ such that $A \subseteq a+\mathbb{R}_{+}^{m}$, then $\operatorname{Min}_{\mathbb{R}_{+}^{m}} A \neq \emptyset$. Indeed, for each sequence $\left\{y_{n}\right\}_{n \in \mathbb{N}}=\left\{\left(y_{n}^{1}, y_{n}^{2}, \ldots, y_{n}^{m}\right)\right\}_{n \in \mathbb{N}}$ in $A$ with $y_{n}-y_{n+1} \in \mathbb{R}_{+}^{m}$, it is easy to see that, for each $j=1,2, \ldots, m,\left\{y_{n}^{j}\right\}_{n \in \mathbb{N}}$ is a decreasing sequence in $\mathbb{R}$ and bounded from below. Then $\| y_{n}-$ $y_{n+1} \| \rightarrow 0$ as $n \rightarrow \infty$ and $\operatorname{Min}_{\mathbb{R}_{+}^{m}} A \neq \emptyset$.

\section{Saddle Point Problems}

Theorem 3.1. Let $\left(X, d_{X}\right)$ and $\left(Y, d_{Y}\right)$ be two complete metric spaces. Assume that

(i) for each $(x, y) \in X \times Y,\{(u, v) \in X \times Y: P(x, v) \cap[P(u, y)+K] \neq \emptyset\}$ is a closed set,

(ii) for each $(x, y),(u, v),(a, b) \in X \times Y$, if $P(x, v) \cap[P(u, y)+K] \neq \emptyset$ and $P(u, b) \cap[P(a, v)+$ $K] \neq \emptyset$, then $P(x, b) \cap[P(a, y)+K] \neq \emptyset$,

(iii) for each sequence $\left\{\left(x_{n}, y_{n}\right)\right\}_{n \in \mathbb{N}}$ in $X \times Y$, if $P\left(x_{n}, y_{n+1}\right) \cap\left[P\left(x_{n+1}, y_{n}\right)+K\right] \neq \emptyset$, then $d_{X}\left(x_{n}, x_{n+1}\right) \rightarrow 0$ and $d_{Y}\left(y_{n}, y_{n+1}\right) \rightarrow 0$ as $n \rightarrow \infty$.

Then, for each $(u, v) \in X \times Y$, there exists $(\bar{x}, \bar{y}) \in X \times Y$ such that $P(u, \bar{y}) \cap[P(\bar{x}, v)+K] \neq \emptyset$, $P(\bar{x}, \bar{y}) \cap \operatorname{Min}_{K} P(X, \bar{y}) \neq \emptyset$, and $P(\bar{x}, \bar{y}) \cap \operatorname{Max}_{K} P(\bar{x}, Y) \neq \emptyset$.

Proof. Let $d_{X \times Y}((x, y),(u, v))=d_{X}(x, u)+d_{Y}(y, v)$. Then $\left(X \times Y, d_{X \times Y}\right)$ is a complete metric space. Take any $(u, v) \in X \times Y$. Define the set $M:=\{(x, y) \in X \times Y: P(u, y) \cap[P(x, v)+$ $K] \neq \emptyset\}$. Clearly, $\left(M, d_{X \times Y}\right)$ is a complete metric space. Let $G: M \times M \multimap Z$ be defined by $G(x, y, u, v)=P(x, v)-P(u, y)-K$. By Theorem 2.7, there exists $(\bar{x}, \bar{y}) \in M$ such that $P(\bar{x}, y) \cap[P(x, \bar{y})+K]=\emptyset$ for all $(x, y) \in M \backslash\{(\bar{x}, \bar{y})\}$. By (ii) and the definition of $M$, it is easy to see that $P(\bar{x}, y) \cap[P(x, \bar{y})+K]=\emptyset$ for all $(x, y) \in X \times Y \backslash\{(\bar{x}, \bar{y})\}$. Hence,

$$
\begin{array}{ll}
P(\bar{x}, y) \cap[P(\bar{x}, \bar{y})+K]=\emptyset \quad \forall y \in Y \backslash\{\bar{y}\}, \\
P(x, \bar{y}) \cap[P(\bar{x}, \bar{y})-K]=\emptyset \quad \forall x \in X \backslash\{\bar{x}\} .
\end{array}
$$

Since $P(\bar{x}, \bar{y})$ is a nonempty compact set, by Theorem 2.5 , there exist $z_{1}, z_{2} \in P(\bar{x}, \bar{y})$ such that $z_{1} \in \operatorname{Min}_{K} P(\bar{x}, \bar{y})$ and $z_{2} \in \operatorname{Max}_{K} P(\bar{x}, \bar{y})$. Next, it is easy to see that $P(\bar{x}, Y) \cap\left[z_{2}+K\right]=\left\{z_{2}\right\}$ and $P(X, \bar{y}) \cap\left[z_{1}-K\right]=\left\{z_{1}\right\}$. Therefore, $z_{2} \in P(\bar{x}, \bar{y}) \cap \operatorname{Max}_{K} P(\bar{x}, Y) \neq \emptyset$ and $z_{1} \in P(\bar{x}, \bar{y}) \cap$ $\operatorname{Min}_{K} P(X, \bar{y}) \neq \emptyset$. 
Remark 3.2. If $P$ is an u.s.c. multivalued map with nonempty compact values, then condition (i) of Theorem 3.1 holds.

Example 3.3. Let $X=[0,1], Y=[1,2], Z=\mathbb{R}^{2}$, and $K=\mathbb{R}_{+}^{2}$. Let $P: X \times Y \multimap Z$ be defined by $P(x, y)=\{(x,-y)\}$ for each $(x, y) \in X \times Y$. By Theorem 3.1, for each $(u, v) \in X \times Y$, there exists $(\bar{x}, \bar{y}) \in X \times Y$ such that $P(u, \bar{y}) \cap[P(\bar{x}, v)+K] \neq \emptyset, P(\bar{x}, \bar{y}) \cap \operatorname{Min}_{K} P(X, \bar{y}) \neq \emptyset$, and $P(\bar{x}, \bar{y}) \cap \operatorname{Max}_{K} P(\bar{x}, Y) \neq \emptyset$. Indeed, for each $(u, v) \in X \times Y,(\bar{x}, \bar{y})=(0,1)$.

In Theorem 3.1, if $Y$ is singleton, then we have the following result.

Corollary 3.4. Let $F: X \multimap Z$ be a multivalued map with nonempty compact values. Assume that

(i) for each $x \in X,\{y \in X: F(x) \cap[F(y)+K] \neq \emptyset\}$ is a closed set,

(ii) for each $x, y, z \in X$, if $F(x) \cap[F(y)+K] \neq \emptyset$ and $F(y) \cap[F(z)+K] \neq \emptyset$, then $F(x) \cap$ $[F(z)+K] \neq \emptyset$,

(iii) for each sequence $\left\{x_{n}\right\}_{n \in \mathbb{N}}$ in $X$, if $F\left(x_{n}\right) \cap\left[F\left(x_{n+1}\right)+K\right] \neq \emptyset$, then $d_{X}\left(x_{n}, x_{n+1}\right) \rightarrow 0$ as $n \rightarrow \infty$.

Then, for each $u \in X$, there exists $\bar{x} \in X$ such that $F(u) \cap[F(\bar{x})+K] \neq \emptyset$ and $F(\bar{x}) \cap \operatorname{Min}_{K} F(X) \neq \emptyset$.

Theorem 3.5. Assume that

(i) for each $(x, y) \in X \times Y,\{(u, v) \in X \times Y: P(x, v) \subseteq P(u, y)+K\}$ is closed,

(ii) for each $(x, y),(u, v),(a, b) \in X \times Y$, if $P(x, v) \subseteq P(u, y)+K$ and $P(u, b) \subseteq P(a, v)+K$, then $P(x, b) \subseteq P(a, y)+K$,

(iii) for each sequence $\left\{\left(x_{n}, y_{n}\right)\right\}_{n \in \mathbb{N}}$ in $X \times Y$, if $P\left(x_{n}, y_{n+1}\right) \subseteq P\left(x_{n+1}, y_{n}\right)+K$, then $d_{X}\left(x_{n}, x_{n+1}\right) \rightarrow 0$ and $d_{Y}\left(y_{n}, y_{n+1}\right) \rightarrow 0$ as $n \rightarrow \infty$.

Then, for each $(u, v) \in X \times Y$, there exists $(\bar{x}, \bar{y}) \in X \times Y$ such that $P(u, \bar{y}) \subseteq P(\bar{x}, v)+K$, and $P(\bar{x}, y) \nsubseteq P(x, \bar{y})+K$ for all $(x, y) \in X \times Y \backslash\{(\bar{x}, \bar{y})\}$.

Proof. Let $d_{X \times Y}((x, y),(u, v))=d_{X}(x, u)+d_{Y}(y, v)$. Take any $(u, v) \in X \times Y$. Define the set $M=\{(x, y) \in X \times Y: P(u, y) \subseteq P(x, v)+K\}$. Clearly, $\left(M, d_{X \times Y}\right)$ is a complete metric space. Let $G: M \times M \multimap Z$ be defined by $G(x, y, u, v)=Z \backslash\{P(x, v)-Z \backslash[P(u, y)+K]\}$. For each $(x, y) \in M$, by $(\mathrm{i}),\{(u, v) \in M: 0 \in G(x, y, u, v)\}=\{(u, v) \in M: P(x, v) \subseteq P(u, y)+K\}$ is a closed set. Then by Theorem 2.7 , there exists $(\bar{x}, \bar{y}) \in M$ such that $P(\bar{x}, y) \nsubseteq P(x, \bar{y})+K$ for all $(x, y) \in M \backslash\{(\bar{x}, \bar{y})\}$.

Now, we want to show that $P(\bar{x}, y) \nsubseteq P(x, \bar{y})+K$ for all $(x, y) \in X \times Y \backslash\{(\bar{x}, \bar{y})\}$. In fact, we only need to consider that $(x, y) \notin M$. Suppose that $P(\bar{x}, y) \subseteq P(x, \bar{y})+K$. By (ii) and $(\bar{x}, \bar{y}) \in M,(x, y) \in M$ and this is a contradiction. Therefore, $P(\bar{x}, y) \nsubseteq P(x, \bar{y})+K$ for all $(x, y) \in X \times Y \backslash\{(\bar{x}, \bar{y})\}$.

Remark 3.6. Condition (i) of Theorem 3.5 can be replaced by $y \multimap P(x, y)$ being 1.s.c. and $x \rightarrow P(x, y)$ being an u.s.c. multivalued map with nonempty compact values.

Example 3.7. Let $X=\{1,2\}, Y=[1,2], Z=\mathbb{R}^{2}$, and $K=\mathbb{R}_{+}^{2}$, and let $P: X \times Y \multimap Z$ be defined by

$$
P(x, y):= \begin{cases}\left\{(1, z) \in \mathbb{R}^{2}: y \leq z \leq 2\right\} & \text { if } x=1 \\ \left\{(2, z) \in \mathbb{R}^{2}: y \leq z \leq 2\right\} & \text { if } x=2\end{cases}
$$

This is an example for Theorem 3.5. 
Example 3.8. Let $X=[0, \infty), Y=(-\infty, 0], Z=\mathbb{R}^{2}$, and $K=\mathbb{R}_{+}^{2}$. Let $P: X \times Y \multimap Z$ be defined by $P(x, y)=[x, \infty) \times[y, \infty)$ for each $(x, y) \in X \times Y$. By Theorem 3.5, for each $(u, v) \in X \times Y$, there exists $(\bar{x}, \bar{y}) \in X \times Y$ such that $P(u, \bar{y}) \subseteq P(\bar{x}, v)+K$, and $P(\bar{x}, y) \nsubseteq P(x, \bar{y})+K$ for all $(x, y) \in X \times Y \backslash\{(\bar{x}, \bar{y})\}$. Indeed, $(\bar{x}, \bar{y})=(0,0)$ for each $(u, v) \in X \times Y$.

\section{Bilevel Problems}

Proposition 4.1. Let $Q: X \times Y \multimap X \times Y$ be defined by

$$
\begin{aligned}
Q(u, v) & =A_{1}(u, v) \cap B_{1} \cap C_{1}, \text { where } \\
A_{1}(u, v) & :=\{(x, y) \in X \times Y: P(u, y) \cap[P(x, v)+K] \neq \emptyset\}, \\
B_{1} & :=\left\{(x, y) \in X \times Y: P(x, y) \cap W_{M i n} P(X, y) \neq \emptyset\right\}, \\
C_{1} & :=\left\{(x, y) \in X \times Y: P(x, y) \cap W_{M^{\prime}} P(x, Y) \neq \emptyset\right\} .
\end{aligned}
$$

If $P$ is a continuous multivalued map with nonempty compact values, then $Q$ is closed, and $B_{1} \cap C_{1}$ is a closed set.

Proof. Here, we only need to show that $Q$ is closed. If $\left\{\left(u_{\alpha}, v_{\alpha}, x_{\alpha}, y_{\alpha}\right)\right\}_{\alpha \in \Lambda} \subseteq \operatorname{Gr}(Q)$ and $\left(u_{\alpha}, v_{\alpha}, x_{\alpha}, y_{\alpha}\right) \rightarrow(u, v, x, y)$, for each $\alpha \in \Lambda$, we have then

(1) $P\left(u_{\alpha}, y_{\alpha}\right) \cap\left[P\left(x_{\alpha}, v_{\alpha}\right)+K\right] \neq \emptyset$,

(2) $P\left(x_{\alpha}, y_{\alpha}\right) \cap \operatorname{WMin}_{K} P\left(X, y_{\alpha}\right) \neq \emptyset$,

(3) $P\left(x_{\alpha}, y_{\alpha}\right) \cap \operatorname{WMax}_{K} P\left(x_{\alpha}, Y\right) \neq \emptyset$.

By (1), for each $\alpha \in \Lambda$, there exists $a_{\alpha} \in P\left(u_{\alpha}, y_{\alpha}\right) \cap\left[P\left(x_{\alpha}, v_{\alpha}\right)+K\right]$ and $b_{\alpha} \in P\left(x_{\alpha}, v_{\alpha}\right)$ such that $a_{\alpha}-b_{\alpha} \in K$. Let $L_{1}:=\left\{x_{\alpha}: \alpha \in \Lambda\right\} \cup\{x\}, L_{2}:=\left\{y_{\alpha}: \alpha \in \Lambda\right\} \cup\{y\}, L_{3}:=\left\{v_{\alpha}: \alpha \in \Lambda\right\} \cup\{v\}$, and $L_{4}:=\left\{u_{\alpha}: \alpha \in \Lambda\right\} \cup\{u\}$. Then $L_{1}, L_{2}, L_{3}$, and $L_{4}$ are compact sets. By Lemma 2.2, $P\left(L_{4} \times L_{2}\right)$ and $P\left(L_{1} \times L_{3}\right)$ are compact sets. Hence, we may assume that $a_{\alpha} \rightarrow a$ and $b_{\alpha} \rightarrow b$. By Lemma 2.2, $a \in P(u, y)$ and $b \in P(x, v)$. Clearly, $a-b \in K$ and $P(u, y) \cap[P(x, v)+K] \neq \emptyset$. By (2), for each $\alpha \in \Lambda$, there exists $d_{\alpha} \in P\left(x_{\alpha}, y_{\alpha}\right) \cap \mathrm{WMin}_{K} P\left(X, y_{\alpha}\right)$. By Lemma 2.2, $P\left(L_{1} \times L_{2}\right)$ is a compact set. Hence, we may assume that $d_{\alpha} \rightarrow d$. By Lemma 2.2, $d \in P(x, y) \subseteq P(X, y)$.

For each $\alpha \in \Lambda, P\left(X, y_{\alpha}\right) \cap\left[d_{\alpha}-\operatorname{int}(K)\right]=\emptyset$. Take any $p \in P(X, y)$. Then there exists $r \in X$ such that $p \in P(r, y)$. There exists a net $\left\{p_{\alpha}\right\}_{\alpha \in \Lambda}$ in $Z$ such that $p_{\alpha} \in P\left(r, y_{\alpha}\right) \subseteq$ $P\left(X, y_{\alpha}\right)$ for all $\alpha \in \Lambda$, and $p_{\alpha} \rightarrow p, p_{\alpha}-d_{\alpha} \notin-\operatorname{int}(K)$ for all $\alpha \in \Lambda$. Therefore, $p-d \notin-\operatorname{int}(K)$ for all $p \in P(X, y)$. That is, $P(X, y) \cap[d-\operatorname{int}(K)]=\emptyset$. Hence, $d \in P(x, y) \cap \mathrm{WMin}_{K} P(X, y) \neq \emptyset$. Similarly, we can prove that $P(x, y) \cap \operatorname{WMax}_{K} P(x, Y) \neq \emptyset$. So, $(u, v, x, y) \in \operatorname{Gr}(Q)$ and $Q$ is closed.

Lemma 4.2. Let $X$ and $Y$ be topological spaces, and let $G: X \multimap Y$ be a multivalued map. Let $G^{-1}: G(X) \multimap X$ be defined by $G^{-1}(y):=\{x \in X: y \in G(x)\}$ for each $y \in G(X)$. If $G^{-1}$ is an u.s.c. multivalued map with nonempty compact values and $A$ is a nonempty closed subset of $X$, then $G(A)$ is a closed subset of $Y$.

Proof. If $y \in \operatorname{cl}(G(A))$, then there exist a net $\left\{y_{\alpha}\right\}_{\alpha \in \Lambda}$ such that $y_{\alpha} \rightarrow y$ and a net $\left\{x_{\alpha}\right\}_{\alpha \in \Lambda}$ in $A$ such that $y_{\alpha} \in G\left(x_{\alpha}\right)$ for all $\alpha \in \Lambda$. Hence, $x_{\alpha} \in G^{-1}\left(y_{\alpha}\right)$ for all $\alpha \in \Lambda$. Let $L=\left\{y_{\alpha}: \alpha \in\right.$ $\Lambda\} \cup\{y\}$. Then $L$ is a compact set and $G^{-1}(L)$ is a compact set. We may assume that $x_{\alpha} \rightarrow x$. 
Since $A$ is a closed set, $x \in A$. By Lemma 2.2, $x \in G^{-1}(y)$ and $y \in G(x) \subseteq G(A)$. Therefore, $G(A)$ is a closed subset of $Y$.

Theorem 4.3. In Theorem 3.1, let $G: X \times Y \multimap W$ be a multivalued map with nonempty values, and further assume that

(a) $G^{-1}$ is an u.s.c. multivalued map with nonempty compact values,

(b) $P$ is a continuous multivalued map with nonempty compact values,

(c) for each sequence $\left\{w_{n}\right\}_{n \in \mathbb{N}}$ in $G(X \times Y)$, if $w_{n}-w_{n+1} \in C$, then $\left\|w_{n}-w_{n+1}\right\| \rightarrow 0$ as $n \rightarrow \infty$.

Then there is a solution of problem (BL-1).

Proof. Let $B_{1}$ and $C_{1}$ be defined as in Proposition 4.1. By Theorem 3.1 and Proposition 4.1, $B_{1} \cap C_{1}$ is a nonempty closed subset of $X \times Y$. By Lemma $4.2, G\left(B_{1} \cap C_{1}\right)$ is a nonempty closed subset of $W$. By Theorem 2.11, there exists $(\bar{x}, \bar{y}) \in B_{1} \cap C_{1}$ such that $G(\bar{x}, \bar{y}) \cap \operatorname{Min}_{c} G\left(B_{1} \cap\right.$ $\left.C_{1}\right) \neq \emptyset$.

Example 4.4. In Example 3.3, let $W=\mathbb{R}^{2}, C=\mathbb{R}_{+}^{2}$, and $G(x, y):=(x, y)+\mathbb{R}_{+}^{2}$ for each $(x, y) \in$ $X \times Y$. Clearly, $G(X \times Y)=(0,1)+\mathbb{R}_{+}^{2}$ is a closed subset of $\mathbb{R}^{2}$. Besides, we have

$$
G^{-1}(u, v):=[0, \min \{u, 1\}] \times[1, \min \{v, 2\}]
$$

and $G^{-1}$ is an u.s.c. multivalued map with nonempty compact values. By Theorem 4.3 , there is a solution of problem (BL-1). Indeed, the solution set is $\{(0,1)\}$.

The following theorem is similar to Theorem 4.3. Note that the conditions of Theorems 4.3 and 4.5 are different.

Theorem 4.5. In Theorem 3.1, let $G: X \times Y \multimap W$ be a multivalued map, and further assume that

(a) $G$ is an u.s.c. multivalued map with nonempty compact values,

(b) for each $x, y, z \in X \times Y$, if $G(x) \cap[G(y)+K] \neq \emptyset$ and $G(y) \cap[G(z)+K] \neq \emptyset$, then $G(x) \cap[G(z)+K] \neq \emptyset$,

(c) $P$ is a continuous multivalued map with nonempty compact values,

(d) for each $\left\{\left(x_{n}\right)\right\}_{n \in \mathbb{N}}$ in $X \times Y$, if $G\left(x_{n}\right) \cap\left[G\left(x_{n+1}\right)+K\right] \neq \emptyset$, then $d_{X \times Y}\left(x_{n}, x_{n+1}\right) \rightarrow 0$ as $n \rightarrow \infty$.

Then there is a solution of problem (BL-1).

Proof. Let $B_{1}$ and $C_{1}$ be defined as in Proposition 4.1. By Theorem 3.1 and Proposition 4.1, $B_{1} \cap C_{1}$ is a nonempty closed subset of $X \times Y$. Hence, $\left(B_{1} \cap C_{1}, d_{X \times Y}\right)$ is a complete metric space. Now, for each $(x, y) \in B_{1} \cap C_{1}$, let $A_{x, y}:=\left\{(u, v) \in B_{1} \cap C_{1}: G(x, y) \cap[G(u, v)+K] \neq \emptyset\right\}$. If $(u, v) \in \operatorname{cl}\left(A_{x, y}\right)$, then there exists a net $\left\{\left(u_{\alpha}, v_{\alpha}\right)\right\}_{\alpha \in \Lambda}$ in $A_{x, y}$ such that $\left(u_{\alpha}, v_{\alpha}\right) \rightarrow(u, v)$. Then for each $\alpha \in \Lambda$, there exists $w_{\alpha} \in G(x, y) \cap\left[G\left(u_{\alpha}, v_{\alpha}\right)+K\right]$. Since $G(x, y)$ is a compact set, we may assume that $w_{\alpha} \rightarrow w \in G(x, y)$. There exists $t_{\alpha} \in G\left(u_{\alpha}, v_{\alpha}\right)$ such that $w_{\alpha}-t_{\alpha} \in K$. Let $L=\left\{\left(u_{\alpha}, v_{\alpha}\right): \alpha \in \Lambda\right\} \cup\{(u, v)\}$. Clearly, $L$ and $G(L)$ are compact sets. Hence, we may assume that $t_{\alpha} \rightarrow t$. By Lemma 2.2, $t \in G(u, v)$. Clearly, $w-t \in K$ and this implies that 
$G(x, y) \cap[G(u, v)+K] \neq \emptyset$. Therefore, $(u, v) \in A_{x, y}$ and $A_{x, y}$ is a closed set. By Corollary 3.4, there exists $(\bar{x}, \bar{y}) \in B_{1} \cap C_{1}$ such that $G(\bar{x}, \bar{y}) \cap \operatorname{Min}_{c} G\left(B_{1} \cap C_{1}\right) \neq \emptyset$.

Furthermore, we have the following result which is different from Theorems 4.3 and 4.5. In Theorem 4.6, $W$ is a Hausdorff t.v.s., and $X$ and $Y$ are compact metric spaces. In Theorems 4.3 and $4.5, W$ is a Banach space, and $X$ and $Y$ are complete metric spaces.

Theorem 4.6. In Theorem 3.1, let $G: X \times Y \multimap W$ be a multivalued map, and further assume that

(a) $G$ is an u.s.c. multivalued map with nonempty compact values,

(b) $P$ is a continuous multivalued map with nonempty compact values,

(c) $X$ and $Y$ are compact.

Then there is a solution of problem (BL-1).

Proposition 4.7. Let $Q: X \times Y \multimap X \times Y$ be defined by

$$
\begin{aligned}
Q(u, v) & :=A_{2}(u, v) \cap B_{2} \cap C_{2}, \text { where } \\
A_{2}(u, v) & :=\{(x, y) \in X \times Y: P(u, y) \subseteq P(x, v)+K\}, \\
B_{2} & :=\{(x, y) \in X \times Y: P(x, y) \nsubseteq P(a, y)+\operatorname{int}(K) \quad \forall a \in X\}, \\
C_{2} & :=\{(x, y) \in X \times Y: P(x, b) \nsubseteq P(x, y)+\operatorname{int}(K) \quad \forall b \in Y\} .
\end{aligned}
$$

If $P$ is a continuous multivalued map with nonempty compact values, then $Q$ is closed, and $B_{2} \cap C_{2}$ is a closed set.

Proof. Here, we only need to show that $Q$ is closed. If $\left\{\left(u_{\alpha}, v_{\alpha}, x_{\alpha}, y_{\alpha}\right)\right\}_{\alpha \in \Lambda} \subseteq \operatorname{Gr}(Q)$ and $\left(u_{\alpha}, v_{\alpha}, x_{\alpha}, y_{\alpha}\right) \rightarrow(u, v, x, y)$, then we have

(1) $P\left(u_{\alpha}, y_{\alpha}\right) \subseteq P\left(x_{\alpha}, v_{\alpha}\right)+K$,

(2) $P\left(x_{\alpha}, y_{\alpha}\right) \nsubseteq P\left(a, y_{\alpha}\right)+\operatorname{int}(K)$ for all $a \in X$,

(3) $P\left(x_{\alpha}, b\right) \nsubseteq P\left(x_{\alpha}, y_{\alpha}\right)+\operatorname{int}(K)$ for all $b \in Y$.

Take any $z \in P(u, y)$; there exists a net $\left\{z_{\alpha}\right\}_{\alpha \in \Lambda}$ such that $z_{\alpha} \in P\left(u_{\alpha}, y_{\alpha}\right)$ for all $\alpha \in \Lambda$ and $z_{\alpha} \rightarrow z$. There exists $w_{\alpha} \in P\left(x_{\alpha}, v_{\alpha}\right)$ such that $z_{\alpha}-w_{\alpha} \in K$. Let $L_{1}:=\left\{x_{\alpha}: \alpha \in \Lambda\right\} \cup\{x\}$, $L_{2}:=\left\{y_{\alpha}: \alpha \in \Lambda\right\} \cup\{y\}, L_{3}:=\left\{v_{\alpha}: \alpha \in \Lambda\right\} \cup\{v\}$, and $L_{4}:=\left\{u_{\alpha}: \alpha \in \Lambda\right\} \cup\{u\}$. Then $L_{1}, L_{2}, L_{3}$, and $L_{4}$ are compact sets and $P\left(L_{1} \times L_{3}\right)$ is a compact set. Hence, we may assume that $w_{\alpha} \rightarrow w$. By Lemma 2.2, $w \in P(x, v)$. Clearly, $z \in w+K$. Hence, $z \in P(x, v)+K$, and $P(u, y) \subseteq P(x, v)+K$.

Take any $a \in X$; there exists $s_{\alpha} \in P\left(x_{\alpha}, y_{\alpha}\right)$ such that $s_{\alpha} \notin P\left(a, y_{\alpha}\right)+\operatorname{int}(K) . P\left(L_{1} \times L_{2}\right)$ is a compact set, and we may assume that $s_{\alpha} \rightarrow s$; By Lemma 2.2, $s \in P(x, y)$. Take any $t \in P(a, y)$, there exists a net $\left\{t_{\alpha}\right\}_{\alpha \in \Lambda}$ such that $t_{\alpha} \in P\left(a, y_{\alpha}\right)$ for all $\alpha \in \Lambda$ and $t_{\alpha} \rightarrow t$, $s_{\alpha}-t_{\alpha} \notin \operatorname{int}(K)$ for all $\alpha \in \Lambda$. Clearly, $s-t \notin \operatorname{int}(K)$ for all $t \in P(a, y)$. Hence, $P(x, y) \nsubseteq P(a, y)+$ $\operatorname{int}(K)$ for all $a \in X$. Similarly, $P(x, b) \nsubseteq P(x, y)+\operatorname{int}(K)$ for all $b \in Y$. Therefore, $Q$ is closed.

Applying Proposition 4.7 and following the similar argument as in the proof of Theorems 4.3-4.6, we can get the following similar results. 
Theorem 4.8. In Theorem 3.5, let $G: X \times Y \multimap W$ be a multivalued map with nonempty values. Further assume that conditions (a)-(c) of Theorem 4.3 (resp., conditions (a)-(d) of Theorem 4.5) are satisfied. Then there is a solution of problem (BL-2).

Proposition 4.9. Let $P: X \times Y \rightarrow \mathbb{R}^{m}$ be a map with $P(x, y)=\left(P_{1}(x, y), P_{2}(x, y), \ldots, P_{m}(x, y)\right)$. Let $Q: X \times Y \multimap X \times Y$ be defined by

$$
\begin{aligned}
Q(u, v) & :=A_{3}(u, v) \cap B_{3} \cap C_{3}, \text { where } \\
A_{3}(u, v) & :=\{(x, y) \in X \times Y: P(u, y)-P(x, v) \in K\}, \\
B_{3} & :=\left\{(x, y) \in X \times Y: P(x, y) \in \operatorname{Max}_{\mathbb{R}_{+}^{m}} P(x, Y)\right\}, \\
C_{3} & :=\left\{(x, y) \in X \times Y: P(x, y) \in \operatorname{Min}_{\mathbb{R}_{+}^{m}} P(X, y)\right\} .
\end{aligned}
$$

Suppose that, for each $i=1,2, \ldots, m, P_{i}: X \times Y \rightarrow \mathbb{R}$ is continuous, $x \rightarrow P_{i}(x, y)$ is one to one, and $y \rightarrow P_{i}(x, y)$ is one to one. Then $Q$ is closed, and $B_{3} \cap C_{3}$ is a closed set.

Proof. Let $\left\{\left(u_{n}, v_{n}, x_{n}, y_{n}\right)\right\}_{n \in \mathbb{N}} \subseteq \operatorname{Gr}(Q)$ and $\left(u_{n}, v_{n}, x_{n}, y_{n}\right) \rightarrow(u, v, x, y)$ as $n \rightarrow \infty$. Then $P\left(u_{n}, y_{n}\right)-P\left(x_{n}, v_{n}\right) \in \mathbb{R}_{+}^{m}, P\left(x_{n}, b\right)-P\left(x_{n}, y_{n}\right) \notin \mathbb{R}_{+}^{m}$ for all $b \in Y \backslash\left\{y_{n}\right\}$, and $P\left(x_{n}, y_{n}\right)-$ $P\left(a, y_{n}\right) \notin \mathbb{R}_{+}^{m}$ for all $a \in X \backslash\left\{x_{n}\right\}$. Since $P$ is continuous, $P(u, y)-P(x, v) \in \mathbb{R}_{+}^{m}$. Furthermore, for each $n \in \mathbb{N}$, we have

$$
P\left(x_{n}, y_{n}\right)-P\left(a, y_{n}\right) \notin \mathbb{R}_{+}^{m} \backslash\{0\} \quad \forall a \in X
$$

Take any $a \in X \backslash\{x\}$. There exists $n_{0} \in \mathbb{N}$ such that $x_{n} \neq a$ for all $n \geq n_{0}$. Furthermore, for each $n \in \mathbb{N}$ with $n \geq n_{0}$, there exists $j=j(n) \in\{1,2, \ldots, m\}$ such that $P_{j}\left(x_{n}, y_{n}\right)-P_{j}\left(a, y_{n}\right)<0$. Indeed, if not, there exists $n \in \mathbb{N}$ with $n \geq n_{0}$ such that $P_{j}\left(x_{n}, y_{n}\right)-P_{j}\left(a, y_{n}\right) \geq 0$ for all $j \in\{1,2, \ldots, m\}$. Since $n \geq n_{0}$ and $x \rightarrow P_{j}(x, y)$ is one to one, $P_{j}\left(x_{n}, y_{n}\right)-P_{j}\left(a, y_{n}\right)>0$ for all $j \in\{1,2, \ldots, m\}$. Hence, $P\left(x_{n}, y_{n}\right)-P\left(a, y_{n}\right) \in \operatorname{int}\left(\mathbb{R}_{+}^{m}\right)$. This leads to a contradiction.

Therefore, there exist $k \in\{1,2, \ldots, m\}$, and $\left\{x_{n_{t}}\right\}_{t \in \mathbb{N}},\left\{y_{n_{t}}\right\}_{t \in \mathbb{N}}$ of $\left\{x_{n}\right\}_{n \in \mathbb{N}}$, and $\left\{y_{n}\right\}_{n \in \mathbb{N}}$, respectively, such that $P_{k}\left(x_{n_{t}}, y_{n_{t}}\right)-P_{k}\left(a, y_{n_{t}}\right)<0$ for all $t \in \mathbb{N}$. Suppose that $P(x, y)-$ $P(a, y) \in \mathbb{R}_{+}^{m}$. Then $P_{j}(x, y)-P_{j}(a, y) \geq 0$ for all $j \in\{1,2, \ldots, m\}, 0 \leq P_{k}(x, y)-P_{k}(a, y)=$ $\lim _{t \rightarrow \infty}\left[P_{k}\left(x_{n_{t}}, y_{n_{t}}\right)-P_{k}\left(a, y_{n_{t}}\right)\right] \leq 0$. Hence, $P_{k}(x, y)=P_{k}(a, y)$. This leads to a contradiction since $x \rightarrow P_{k}(x, y)$ is one to one. Therefore, $P(x, y)-P(a, y) \notin \mathbb{R}_{+}^{m}$ for all $a \in X \backslash\{x\}$. Similarly, we have $P(x, b)-P(x, y) \notin \mathbb{R}_{+}^{m}$ for all $b \in Y \backslash\{y\}$. Therefore, $Q$ is closed.

Theorem 4.10. Let $P: X \times Y \rightarrow \mathbb{R}^{m}$ be a map with $P(x, y)=\left(P_{1}(x, y), P_{2}(x, y), \ldots, P_{m}(x, y)\right)$. Let $G: X \times Y \multimap W$ be a multivalued map with nonempty values. Further assume that

(i) for each $i=1,2, \ldots, m, P_{i}: X \times Y \rightarrow \mathbb{R}$ is continuous, $x \rightarrow P_{i}(x, y)$ is one to one, and $y \rightarrow P_{i}(x, y)$ is one to one,

(ii) for each $(x, y),(u, v),(a, b) \in X \times Y$, if $P(x, v)-P(u, y) \in \mathbb{R}_{+}^{m}$ and $P(u, b)-P(a, v) \in \mathbb{R}_{+}^{m}$, then $P(x, b)-P(a, y) \in \mathbb{R}_{+}^{m}$,

(iii) for each sequence $\left\{\left(x_{n}, y_{n}\right)\right\}_{n \in \mathbb{N}}$ in $X \times Y$ with $P\left(x_{n}, y_{n+1}\right)-P\left(x_{n+1}, y_{n}\right) \in \mathbb{R}_{+}^{m}$, $d_{X}\left(x_{n}, x_{n+1}\right) \rightarrow 0$ and $d_{Y}\left(y_{n}, y_{n+1}\right) \rightarrow 0$ as $n \rightarrow \infty$, 
(iv) $G^{-1}$ is an u.s.c. multivalued map with nonempty compact values,

(v) for each sequence $\left\{w_{n}\right\}_{n \in \mathbb{N}}$ in $G(X \times Y)$, if $w_{n}-w_{n+1} \in C$, then $\left\|w_{n}-w_{n+1}\right\| \rightarrow 0$ as $n \rightarrow \infty$.

Then there is a solution of problem (BL-3).

Proof. Applying Proposition 4.9 and following the similar argument as in the proof of Theorem 4.3, we can get the proof of Theorem 4.10.

Remark 4.11. The conditions of Theorem 4.10 and Theorem 3.5 in Liou et al. [5] are different. Note that Liou et al. [5] assumed that the feasible set $S_{p}(x)$ is nonempty, and let the considered multivalued map $F$ be proper, lower semicontinuous, and weakly coercive on $\operatorname{Gr}\left(S_{p}\right)$.

\section{Equilibrium Problems and Mathematical Program with Equilibrium Constraint on Complete Metric Spaces}

Theorem 5.1. Let $X$ and $Y$ be a complete metric spaces, and let $H: X \times Y \times X \multimap Z$ be a multivalued map. Assume that

(i) for each $(x, y) \in X \times Y, 0 \in H(x, y, x)$,

(ii) for each $(x, y) \in X \times Y,\{u \in X: 0 \in H(x, y, u)\}$ is a closed subset of $X$,

(iii) for each $x, u, a \in X$ and $y, v \in Y$, if $0 \in H(x, y, u)$ and $0 \in H(u, v, a)$, then $0 \in$ $H(x, y, a)$,

(iv) for each sequence $\left\{\left(x_{n}, y_{n}\right)\right\}_{n \in \mathbb{N}}$ in $X \times Y$ with $0 \in H\left(x_{n}, y_{n}, x_{n+1}\right), d_{X}\left(x_{n}, x_{n+1}\right) \rightarrow 0$ and $d_{Y}\left(y_{n}, y_{n+1}\right) \rightarrow 0$ as $n \rightarrow \infty$.

Then there exists $(\bar{x}, \bar{y}) \in X \times Y$ such that $0 \notin H(\bar{x}, \bar{y}, u)$ for all $u \in X \backslash\{\bar{x}\}$.

Proof. Let $G: X \times Y \times X \times Y \multimap Z$ be defined by $G(x, y, u, v)=H(x, y, u)$ for each $(x, y, u, v) \in$ $X \times Y \times X \times Y$. Then Theorem 5.1 follows from Theorem 2.7.

Theorem 5.2. Let $X$ and $Y$ be a complete metric spaces, and let $S: X \times Y \times X \multimap Z$ be a multivalued map. Assume that

(i) $Z \backslash[K \backslash\{0\}]$ is a closed set; and for each $(x, y) \in X \times Y, S(x, y, x)=\{0\}$,

(ii) for each $(x, y) \in X \times Y,\{u \in X: S(x, y, u) \cap K \neq \emptyset\}$ is a closed subset of $X$,

(iii) for each $x, u, a \in X$ and $y, v \in Y$, if $S(x, y, u) \cap K \neq \emptyset$ and $S(u, v, a) \cap K \neq \emptyset$, then $S(x, y, a) \cap K \neq \emptyset$,

(iv) for each sequence $\left\{\left(x_{n}, y_{n}\right)\right\}_{n \in \mathbb{N}}$ in $X \times Y$ with $S\left(x_{n}, y_{n}, x_{n+1}\right) \cap K \neq \emptyset, d_{X}\left(x_{n}, x_{n+1}\right) \rightarrow 0$ and $d_{Y}\left(y_{n}, y_{n+1}\right) \rightarrow 0$ as $n \rightarrow \infty$,

(v) $G$ is closed; and for each $u \in X,(x, y) \multimap S(x, y, u)$ is l.s.c.,

(vi) for each sequence $\left\{\left(x_{n}, y_{n}, w_{n}\right)\right\}_{n \in \mathbb{N}}$ in $\operatorname{Gr}(G)$ with $w_{n}-w_{n+1} \in C, d_{X \times Y}\left(\left(x_{n}, y_{n}\right)\right.$, $\left.\left(x_{n+1}, y_{n+1}\right)\right) \rightarrow 0$ and $\left\|w_{n}-w_{n+1}\right\| \rightarrow 0$ as $n \rightarrow \infty$.

Then there is a solution of problem (MPEC-1). 
Proof. By Theorem 5.1, there exists $(\bar{x}, \bar{y}) \in X \times Y$ such that $S(\bar{x}, \bar{y}, u) \cap K=\emptyset$ for all $u \in X \backslash\{\bar{x}\}$. Since $S(\bar{x}, \bar{y}, \bar{x})=\{0\}, S(\bar{x}, \bar{y}, u) \cap[K \backslash\{0\}]=\emptyset$ for all $u \in X$.

Let $L_{1}=\{(x, y) \in X \times Y: S(x, y, u) \cap[K \backslash\{0\}]=\emptyset$ for all $u \in X\}$. Clearly, $(\bar{x}, \bar{y}) \in L_{1} \neq \emptyset$. If $(x, y) \in \operatorname{cl}\left(L_{1}\right)$, then there exists a net $\left\{\left(x_{\alpha}, y_{\alpha}\right)\right\}_{\alpha \in \Gamma}$ in $L_{1}$ such that $\left(x_{\alpha}, y_{\alpha}\right) \rightarrow(x, y)$. Then, for each $u \in X, S\left(x_{\alpha}, y_{\alpha}, u\right) \cap[K \backslash\{0\}]=\emptyset$ for all $\alpha \in \Gamma$. That is, $S\left(x_{\alpha}, y_{\alpha}, u\right) \subseteq Z \backslash[K \backslash$ $\{0\}]$ for all $\alpha \in \Gamma$. Take any $u \in X$ and any $z \in S(x, y, u)$; there exists a net $\left\{z_{\alpha}\right\}_{\alpha \in \Gamma}$ such that $z_{\alpha} \in S\left(x_{\alpha}, y_{\alpha}, u\right) \subseteq Z \backslash[K \backslash\{0\}]$ for all $\alpha \in \Gamma$ and $z_{\alpha} \rightarrow z$. Clearly, $z \in Z \backslash[K \backslash\{0\}]$. Hence, $S(x, y, u) \cap[K \backslash\{0\}]=\emptyset$ for all $u \in X$. Then $L_{1}$ is a closed subset of a complete metric space $X \times Y$. Furthermore, $\left(L_{1}, d_{X \times Y}\right)$ is a complete metric space. Let $G_{1}=\left.G\right|_{L_{1}}$. Then $\operatorname{Gr}\left(G_{1}\right)=\left\{(x, y, w) \in X \times Y \times W:(x, y) \in L_{1}, w \in G(x, y)\right\}$ is a closed subset of $X \times Y \times W$. By Theorem 2.8, there is a solution of problem (MPEC-1).

Theorem 5.3. Let $X$ and $Y$ be a complete metric spaces, and let $S: X \times Y \times X \multimap Z$ be a multivalued map. Assume that

(i) for each $(x, y) \in X \times Y, S(x, y, x)=\{0\}$,

(ii) for each $(x, y) \in X \times Y,\{u \in X: S(x, y, u) \subseteq K\}$ is a closed subset of $X$,

(iii) for each $x, u, a \in X$ and $y, v \in Y$, if $S(x, y, u) \subseteq K$ and $S(u, v, a) \subseteq K$, then $S(x, y, a) \subseteq$ $K$,

(iv) for each sequence $\left\{\left(x_{n}, y_{n}\right)\right\}_{n \in \mathbb{N}}$ in $X \times Y$ with $S\left(x_{n}, y_{n}, x_{n+1}\right) \subseteq K, d_{X}\left(x_{n}, x_{n+1}\right) \rightarrow 0$ and $d_{Y}\left(y_{n}, y_{n+1}\right) \rightarrow 0$ as $n \rightarrow \infty$.,

(v) $Z \backslash[K \backslash\{0\}]$ is a closed set,

(vi) for each $u \in X,(x, y) \multimap S(x, y, u)$ is an u.s.c. multivalued map with nonempty compact values,

(vii) $G$ is closed,

(viii) for each sequence $\left\{\left(x_{n}, y_{n}, w_{n}\right)\right\}_{n \in \mathbb{N}}$ in $\operatorname{Gr}(G)$ with $w_{n}-w_{n+1} \in C, d_{X \times Y}\left(\left(x_{n}, y_{n}\right)\right.$, $\left.\left(x_{n+1}, y_{n+1}\right)\right) \rightarrow 0$ and $\left\|w_{n}-w_{n+1}\right\| \rightarrow 0$ as $n \rightarrow \infty$.

Then there is a solution of problem (MPEC-2).

Proof. Let $H$ be defined by $H(x, y, u)=Z \backslash[S(x, y, u)-[Z \backslash K]]$ for each $(x, y, u) \in X \times Y \times X$. By Theorem 5.1 and condition (i), there exists $(\bar{x}, \bar{y}) \in X \times Y$ such that $S(\bar{x}, \bar{y}, u) \nsubseteq K \backslash\{0\}$ for all $u \in X$.

Let $L_{3}=\{(x, y) \in X \times Y: S(x, y, u) \nsubseteq K \backslash\{0\}$ for all $u \in X\}$. If $(x, y) \in \operatorname{cl}\left(L_{3}\right)$, then there exists a sequence $\left\{\left(x_{n}, y_{n}\right)\right\}_{n \in \mathbb{N}}$ in $L_{3}$ such that $\left(x_{n}, y_{n}\right) \rightarrow(x, y)$. Let $A=$ $\left\{\left(x_{n}, y_{n}\right)\right\}_{n \in \mathbb{N}} \cup\{(x, y)\}$. Then $A$ is a compact set. For each $n \in \mathbb{N}$, since $\left(x_{n}, y_{n}\right) \in L_{3}$, $S\left(x_{n}, y_{n}, u\right) \nsubseteq K \backslash\{0\}$ for all $u \in X$. For each $u \in X$ and $n \in \mathbb{N}$, there exists $z_{n} \in Z$ such that $z_{n} \in S\left(x_{n}, y_{n}, u\right)$ and $z_{n} \notin K \backslash\{0\}$. Then $\left\{z_{n}\right\}_{n \in \mathbb{N}} \subseteq S(A, u)$. By (vi), we may assume that $z_{n} \rightarrow z \in S(x, y, u)$. Since $Z \backslash[K \backslash\{0\}]$ is a closed set, $z \in Z \backslash[K \backslash\{0\}]$. Hence, $(x, y) \in L_{3}$ and $L_{3}$ is closed. Following the similar argument as in the last part of the proof of Theorem 5.2, we get the proof of Theorem 5.3.

\section{References}

[1] Z.-Q. Luo, J.-S. Pang, and D. Ralph, Mathematical Programs with Equilibrium Constraints, Cambridge University Press, Cambridge, UK, 1996. 
[2] O. Stein and G. Still, "On generalized semi-infinite optimization and bilevel optimization," European Journal of Operational Research, vol. 142, no. 3, pp. 444-462, 2002.

[3] O. Stein, Bilevel Strategies in Semi-Infinite Programming, vol. 71 of Nonconvex Optimization and Its Applications, Kluwer Academic Publishers, Boston, Mass, USA, 2003.

[4] S.. İ. Birbil, G. Bouza, J. B. G. Frenk, and G. Still, "Equilibrium constrained optimization problems," European Journal of Operational Research, vol. 169, no. 3, pp. 1108-1127, 2006.

[5] Y. C. Liou, X. Q. Yang, and J. C. Yao, "Mathematical programs with vector optimization constraints," Journal of Optimization Theory and Applications, vol. 126, no. 2, pp. 345-355, 2005.

[6] L.-J. Lin and G. Still, "Mathematical programs with equilibrium constraints: the existence of feasible point," Optimization, vol. 55, no. 3, pp. 205-219, 2006.

[7] L.-J. Lin, "Mathematical programming with system of equilibrium constraints," Journal of Global Optimization, vol. 37, no. 2, pp. 275-286, 2007.

[8] L.-J. Lin and H.-W. Hsu, "Existences theorems of systems of vector quasi-equilibrium problems and mathematical programs with equilibrium constraint," Journal of Global Optimization, vol. 37, no. 2, pp. 195-213, 2007.

[9] D. T. Luc and C. Vargas, "A saddlepoint theorem for set-valued maps," Nonlinear Analysis: Theory, Methods \& Applications, vol. 18, no. 1, pp. 1-7, 1992.

[10] T. Tanaka, "Generalized quasiconvexities, cone saddle points, and minimax theorem for vectorvalued functions," Journal of Optimization Theory and Applications, vol. 81, no. 2, pp. 355-377, 1994.

[11] I.-S. Kim and Y.-T. Kim, "Loose saddle points of set-valued maps in topological vector spaces," Applied Mathematics Letters, vol. 12, no. 8, pp. 21-26, 1999.

[12] L.-J. Lin, "System of coincidence theorems with applications," Journal of Mathematical Analysis and Applications, vol. 285, no. 2, pp. 408-418, 2003.

[13] Q. H. Ansari, L. J. Lin, and L. B. Su, "Systems of simultaneous generalized vector quasiequilibrium problems and their applications," Journal of Optimization Theory and Applications, vol. 127, no. 1, pp. 27-44, 2005.

[14] L.-J. Lin, "Existence theorems of simultaneous equilibrium problems and generalized vector quasisaddle points," Journal of Global Optimization, vol. 32, no. 4, pp. 613-632, 2005.

[15] J. Y. Fu, "Stampacchia generalized vector quasiequilibrium problems and vector saddle points," Journal of Optimization Theory and Applications, vol. 128, no. 3, pp. 605-619, 2006.

[16] K. Kimura and T. Tanaka, "Existence theorem of cone saddle-points applying a nonlinear scalarization," Taiwanese Journal of Mathematics, vol. 10, no. 2, pp. 563-571, 2006.

[17] X.-H. Gong, "The strong minimax theorem and strong saddle points of vector-valued functions," Nonlinear Analysis: Theory, Methods \& Applications, vol. 68, no. 8, pp. 2228-2241, 2008.

[18] N. X. Tan, "Quasivariational inequalities in topological linear locally convex Hausdorff spaces," Mathematische Nachrichten, vol. 122, pp. 231-245, 1985.

[19] J. P. Aubin and A. Cellina, Differential Inclusion, Springer, Berlin, Germany, 1994.

[20] D. T. Luc, "An existence theorem in vector optimization," Mathematics of Operations Research, vol. 14, no. 4, pp. 693-699, 1989.

[21] L.-G. Huang and X. Zhang, "Cone metric spaces and fixed point theorems of contractive mappings," Journal of Mathematical Analysis and Applications, vol. 332, no. 2, pp. 1468-1476, 2007.

[22] L.-J. Lin and C.-S. Chuang, "Existence theorems for variational inclusion problems and the set-valued vector Ekeland variational principle in a complete metric space," Nonlinear Analysis: Theory, Methods E Applications, vol. 70, no. 7, pp. 2665-2672, 2009. 